\title{
Dispersion managed self-similar parabolic pulses
}

\author{
C FINOT \\ Institut Carnot de Bourgogne, UMR 5029 CNRS-Université de Bourgogne, 9 Av. A. Savary, \\ Dijon, France \\ E-mail : christophe.finot@u-bourgogne.fr
}

\begin{abstract}
We describe the propagation of a parabolic self-similar pulse in an anomalous dispersive nonlinear fibre. Given the capacity of a linearly chirped parabolic pulse to maintain unaffected its typical shape over a short propagation distance, we introduce the concept of dispersion-managed self-similar pulses and outline potential benefits in terms of spectral broadening enhancement.
\end{abstract}

Keywords. Parabolic pulses, nonlinear propagation, nonlinear fibre

\section{Introduction}

Since their first experimental demonstration in 2000 in normally dispersive fibre amplifiers [1], the unique properties of optical parabolic pulses have generated a great deal of attention. Indeed, irrespective to its initial shape, any pulse progressively evolves towards a parabolic intensity profile combined with a strictly linear chirp [2]. This typical shape experiences then a self-similar evolution, with exponential spectral and temporal broadenings and remarkably resists to the deleterious effects of the optical wave-breaking [3, 4].

Several methods have been successfully tested to generate parabolic pulses : rare-earth doped fibres (ytterbium [1, 5] or erbium [6, 7] ), Raman amplifiers [8] as well as a wide set of attractive passive means such as superstructured fibre Bragg gratings [4], a set of two carefully chosen fibres [9], comblike fibre [10] or dispersion decreasing fibre [11]. The experimental interest has strongly been driven by the possibility to fully cancel the linear chirp and thus to generate high-power ultrashort pulses with noticeably low substructures $[1,4-6,12,13]$. Other applications in the telecommunication field such as multiwavelength sources [4] have also taken advantage of the broadened smooth spectrum of parabolic pulses [2].

Previous works have reported the role played by the dispersive properties of the fibre and arrangements of fibres with various normal dispersion values have been suggested to efficiently generate parabolic pulses [9-11]. The purpose of the present article is to extend the concept of parabolic similaritons to a combination of fibres with alternating negative and positive dispersions. Based on dispersion management, a concept that has found impressive applications in high bit-rate optical transmissions [14-16], we demonstrate that a parabolic pulse can maintain its unique features even in presence of anomalous dispersion and nonlinearity.

This article is thus organized as follows. We first describe the model we use and the parameters chosen to characterize the pulse evolution. We then focus on the propagation of parabolic pulses in the 
anomalous regime, with or without nonlinearity. Evolution in dispersion managed fibres is discussed and parabolic typical behaviour is compared with other pulse shapes. Finally, we investigate the influence of the average dispersion.

\section{Model}

The evolution of the complex electrical field $\psi(z, T)$ of a pulse propagating in a passive optical fibre in presence of second order dispersion $\beta_{2}$ and Kerr nonlinearity $\gamma$ can be described by the well-known scalar nonlinear Schrödinger equation (NLSE) [17] :

$$
i \frac{\partial \psi}{\partial z}=\frac{\beta_{2}}{2} \frac{\partial^{2} \psi}{\partial t^{2}}-\gamma|\psi|^{2} \psi
$$

with $z$ the propagation distance and $T$ the time in a copropagating frame.

For the sake of simplicity, we do not include in the present discussion the effects of higher-order linear and nonlinear effects such as third order dispersion, linear attenuation or Raman scattering [17].

We study the evolution of an initial parabolic pulse $\psi_{p}$ having the following characteristic profile :

$$
\begin{cases}\psi_{p}(t)=\sqrt{P_{p}} \sqrt{1-\frac{t^{2}}{T_{p}^{2}}} \exp \left(-i \frac{C_{p}}{2} t^{2}\right) & \text { if }|t| \leq T_{p} \\ \psi_{p}(t)=0 & \text { if }|t|>T_{p}\end{cases}
$$

with $T_{p}, P_{p}$ and $C_{p}$ the temporal width, the peak power and the temporal chirp coefficient of the parabolic pulse respectively [1,2]. As long as the chirp coefficient $C_{p}$ is high, given the stationary phase theorem, the spectral shape $\widetilde{\psi_{p}}(\omega)$ reflects the temporal profile and is then parabolic and linearly chirped [18] :

$$
{\widetilde{\psi_{p}}}_{p}(\omega) \simeq \sqrt{\frac{2 \pi P_{p}}{C_{p}}} \sqrt{1-\frac{\omega^{2}}{\omega_{p}^{2}}} \exp \left(i\left(\frac{\pi}{4}+\frac{\widetilde{C_{p}}}{2} \omega^{2}\right)\right)
$$

with $\omega_{p}$ and $\widetilde{C_{p}}$ the spectral width and chirp coefficient respectively given by:

$$
\omega_{p}=C_{p} T_{p} \quad \text { and } \quad \widetilde{C_{p}}=1 / C_{p}
$$

Equation (1) will be numerically integrated by using the standard split-step Fourier method [17]. In order to avoid some numerical artefacts due to the unphysical compact nature of the parabolic pulse, we have used a slight temporal apodization window based on a $5^{\text {th }}$-order Gaussian shape [4] with a full width at half maximum $(F W H M)$ of $1.6 T_{p}$. We have carefully checked that this apodization window does not influence our results.

During the propagation in the fibre, the pulse will be affected by the combined effects of non-linearity and dispersion. To qualitatively illustrate the pulse dynamics and similarly to the split-step Fourier algorithm, let us consider non-linearity and dispersion acting separately on an infinitesimal length $\delta z$. On the one hand, second order dispersion alone induces in the spectral domain an additional chirp given by $\delta \widetilde{C_{p}}=\beta_{2} \delta z$. Given the relationship (3), the parabolic shape is maintained and the dispersion leads in the temporal domain to new chirp coefficient $C^{\prime}{ }_{p}$, peak power $P^{\prime}{ }_{p}$ and temporal width $T_{p}{ }_{p}$ :

$$
P_{p}^{\prime}=\frac{P_{p}}{1+\beta_{2} C_{p} \delta z}, \quad T_{p}^{\prime}=\left(\begin{array}{lllll}
1+\beta_{2} & C_{p} & \delta z
\end{array}\right) \quad T_{p} \quad \text { and } \quad C_{p}^{\prime}=\frac{C_{p}}{\sqrt{1+\beta_{2} C_{p} \delta z}} .
$$

On the second hand, self-phase modulation (SPM) alone as defined in this model does not affect the temporal intensity profile but results in an additional temporal linear chirp given by $[12,17]$ :

$$
\delta C_{p}=\gamma \quad \delta z \quad P_{p}^{\prime} / T_{p}^{\prime 2}
$$


We can then see that the evolution highly depends on the dispersion regime. For normal dispersion, nonlinearity and dispersion act conjointly to increase the chirp coefficient $C^{\prime}{ }_{p}+\delta C_{p}$ and the temporal width of the pulse increases. On the contrary, it is clear from (5) that the pulse will shorten in the anomalous dispersion regime.

In order to more rigorously predict the evolution of the pulse parameters, the following set of coupled equations can be used $[3,12]$ :

$$
\left\{\begin{array}{l}
\frac{\partial^{2} T_{p}}{\partial z^{2}}=2 T_{p 0} P_{p 0} \frac{\gamma \beta_{2}}{T_{p}^{2}} \\
C_{p}=\frac{1}{\beta_{2} T_{p}} \frac{\partial T_{p}}{\partial z} \\
T_{p 0} P_{p 0}=T_{p} P_{p}
\end{array}\right.
$$

with $T_{p 0}, P_{p 0}$ and $C_{p 0}$ the initial pulse properties.

Equations (7) have been derived from NLSE and implicitly assume that the parabolic typical profile is maintained over the propagation. In order to check this assumption, we characterize the pulse shape thanks to the misfit factor between the pulse intensity and the apodized parabolic pulse $\psi_{p_{-} f t}[12,18]$

$$
M^{2}=\int\left[|\psi|^{2}-\left|\psi_{p_{-} f i t}\right|^{2}\right]^{2} d t / \int|\psi|^{4} d t
$$

To illustrate this article, we study an initial parabolic pulse obtained for example in an erbium doped similariton amplifier at telecommunication wavelengths [6] and having the following properties : $2 T_{p 0}=5 \mathrm{ps}, P_{p 0}=500 \mathrm{~W}$ and $C_{p 0}=1.27$ THz. $\mathrm{ps}^{-1}$. We consider the evolution in passive fibres with a nonlinearity $\gamma=1.510^{-3} \mathrm{~W}^{-1} \cdot \mathrm{m}^{-1}$. Both dispersion regimes will be investigated with fibres having dispersion values of $\beta_{21}=-1010^{-3} \mathrm{ps}^{2} \cdot \mathrm{m}^{-1}$ (fibre 1) and $\beta_{22}=1010^{-3} \mathrm{ps}^{2} \cdot \mathrm{m}^{-1}$ (fibre 2). Such fibre parameters are typical of non-zero dispersion-shifted fibres [19] with reduced dispersion slope. Let us add that the following results could also be demonstrated at other wavelengths such as $1.06 \mu \mathrm{m}$ where high power parabolic pulse generation has been successfully reported [1,5]. Indeed, taking advantage of the progresses in microstructured design and fabrication techniques, using photonic crystal fibres [20] is conceivable with the additional potential benefit of a reduced third-order dispersion.

\section{Parabolic pulse propagating in a single passive fibre}

We first examine the behaviour of a parabolic pulse in a single 15-m fibre. Let us recall that in the case of a single normally dispersive fibre (fibre 2), the parabolic pulse undergoes a temporal broadening illustrated in figure 1(a1). We can also make out that the increase of the temporal width (figure 1(b), solid black line) is accurately described by (7) and that the parabolic shape is kept unaffected as highlighted by the low misfit factor seen in figure 1(c) [3, 9]. A negative initial chirp parameter would have lead to a different dynamics, with a noticeable spectral compression stage [12, 21].

In an anomalous medium, the evolution is rather different. Let us consider the evolution in absence of nonlinearity, as it can happen when using a pair of linear diffraction gratings [1,5], a hollow core photonic bandgap fibre $[6,20]$ or a strongly attenuated pulse $[4,13]$. As it can be seen in figure 1(a2) and figure 1(b) (results obtained with fibre 1, dotted line), the pulse undergoes a temporal compression leading to a transformed limited pulse at a distance $z_{c}$ given by :

$$
z_{c}=\frac{1}{C_{P 0}\left|\beta_{2 A}\right|}
$$

with $\beta_{2 A}$ the second order dispersion of the anomalous medium.

At the optimum compression stage, the shape is not parabolic anymore but becomes a Bessel function of the first kind $[12,13,18]$, which explains the significant increase of the misfit factor. Following this compression stage, the pulse undergoes temporal broadening. In order to better outline this 
broadening, we have also plotted in figure 1(b) the evolution of a pulse having a higher initial chirp coefficient $C^{\prime}{ }_{p 0}$ of 2.56 THz.ps ${ }^{-1}$ : according to (9), the compression occurs faster and we can note that after the compression stage, the pulse broadens again and regains its typical parabolic intensity profile. Indeed, as in a purely dispersive media the pulse spectrum does not change, the relationship between equations (2) and (3) is still valid in the limit of a highly anomalous chirp.
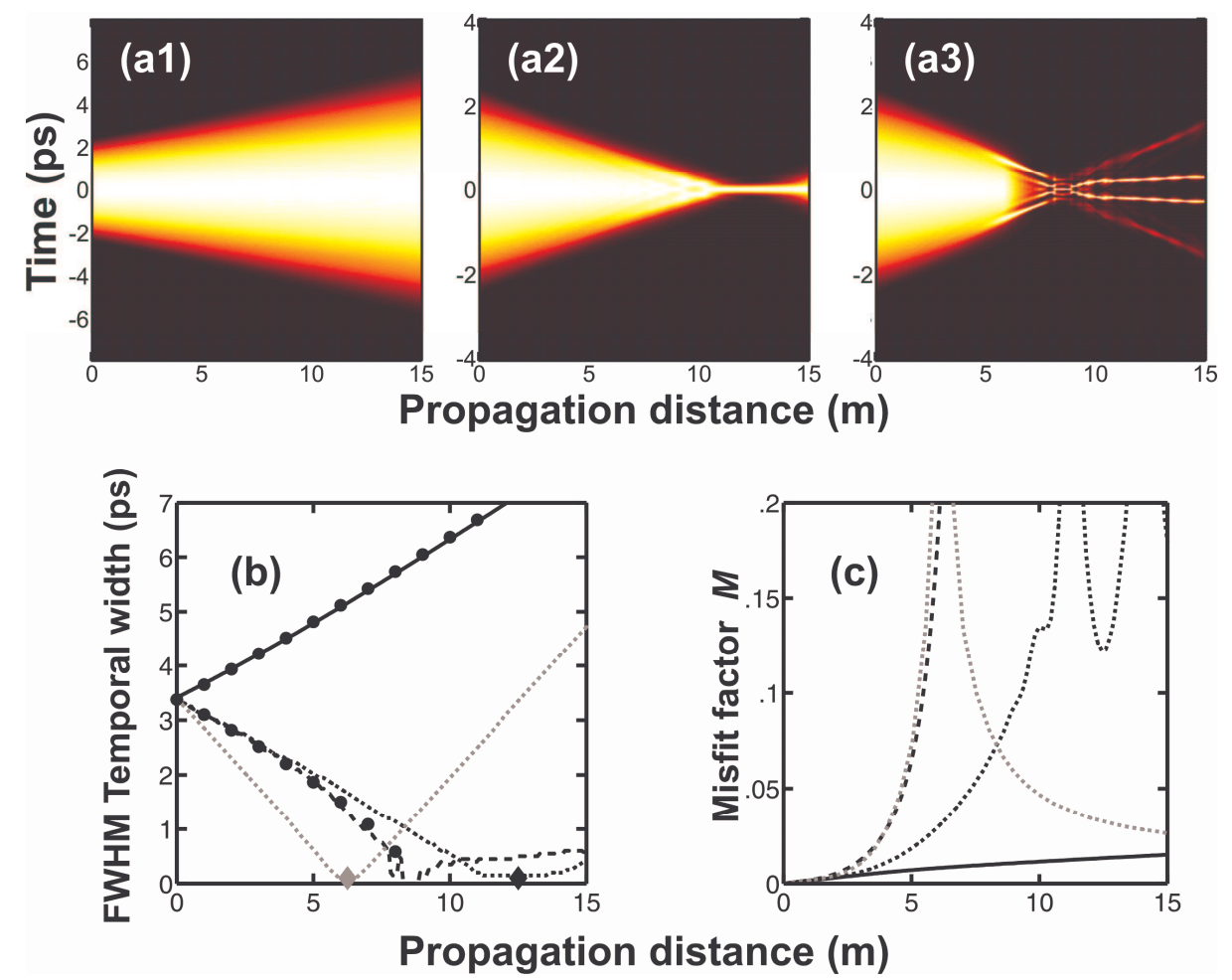

Figure 1: (a) Evolution of the intensity profile with respect to the propagation distance in the case of a parabolic pulse propagating in a normally dispersive nonlinear fibre (fibre 2) (a1), in an anomalous dispersive fibre (fibre 1) without (a2) and with (a3) nonlinearity. Evolution of the FWHM temporal duration of the pulse (b) and of the misfit parameter $M$ (c) of a pulse propagating in a normal dispersive nonlinear fibre (solid line), in an anomalous dispersive fibre without (dotted line) and with (dashed line) nonlinearity. Results obtained for an initial chirp coefficient of $C_{p 0}=1.27$ THz.ps ${ }^{-1}$ and $C_{p 0}^{\prime}=2.56$ THz.ps ${ }^{-1}$ are plotted in black and grey respectively. Results predicted by (7) are plotted with circles. Optimal compression distance as predicted by (9) is indicated by full diamonds.

Let us now evaluate the impact of Kerr nonlinearity (with the chirp parameter $C_{p 0}=1.27 \mathrm{THz} . \mathrm{ps}^{-1}$ ). On the first stage (below $3 \mathrm{~m}$ ), the pulse evolution is similar to the compression obtained without nonlinearity : the parabolic pulse shortens and maintains its shape as assessed by the low misfit factor. A direct consequence of the temporal compression is a peak power enhancement so that nonlinear effects have an increasing role. This results in a rather different evolution as it can be visualized in figure 1(a3). Indeed, the dynamics of the pulse is severely impaired by soliton compression and pulse break-up $[17,22]$ : the temporal compression of the pulse is faster than in the case of a purely dispersive propagation and more remarkably, the pulse splits into four pulses evolving with various velocities. Parabolic pulse approximation becomes obviously completely out of context and contrary to a purely dispersive fibre, the parabolic shape is not regained after further propagation. One can also notice that the best recompressed pulse is achieved in a nonlinearity free fibre, whereas in presence of nonlinearity, much higher sidelobes are present $[18,23]$. 


\section{Parabolic pulse propagation in a dispersion-managed fibre}

In the preceding section, we have pointed out that parabolic pulses evolving in an anomalous medium in presence of nonlinearity were able to maintain their parabolic features only upon a reduced distance. We now study the evolution of the pulse in a fibre with anomalous dispersion (fibre 1) followed by a normally dispersive one (fibre 2). Both fibres have the same length (3 meters) so that the average dispersion of this map (map 1) is null.

Temporal and spectral evolutions are plotted in figure 2(a,b) respectively. After the temporal compression stage described in part 3, the pulse undergoes both temporal and spectral broadening in the second segment. Note that the pulse maintains a parabolic intensity profile as well as a high linear chirp during the whole propagation. The initial and final profiles are compared in figure 2(c-d). Whereas the temporal intensity profile does not exhibit significant changes, the most striking point is that due to SPM, the pulse spectrum has noticeably broadened. This confirms that, as the initial and final properties are not identical, parabolic pulses are intrinsically different from analytical stationary solutions such as dispersion managed solitons [16] or chirped solitons observed in an all-normal dispersion fibre lasers [24].
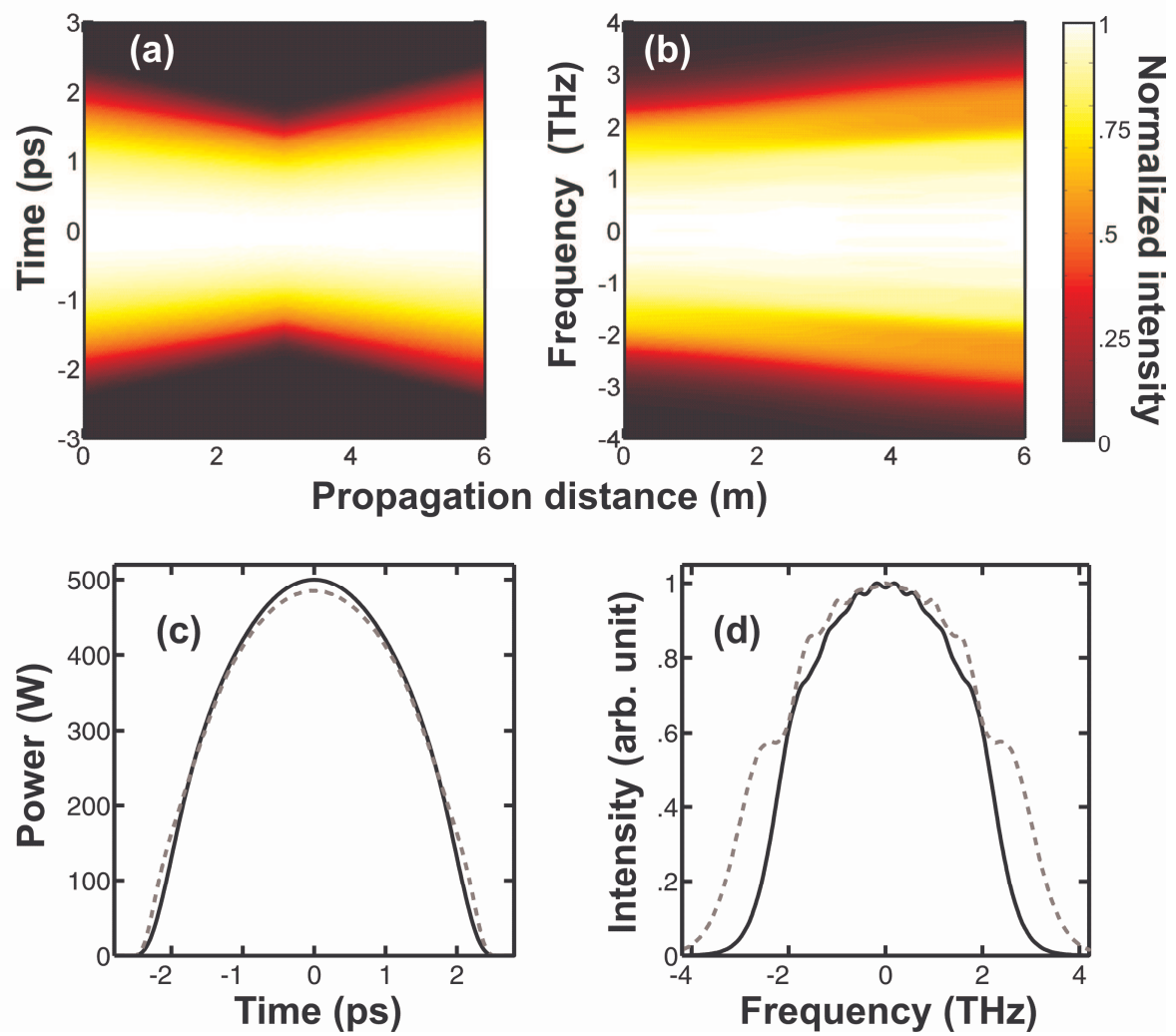

Figure 2 : Longitudinal evolution of the temporal (a) and spectral (b) profiles of a parabolic pulse in a set of two fibres (map1). Initial (solid line) and final (dashed line) temporal (c) and spectral (d) profiles are compared.

Let us now investigate the evolution in a concatenation of the preceding map. The propagation over four maps is displayed in figure 3(a). The evolution is parabolic over the first three maps, but the progressive growth of the misfit factor (figure $3(b)$ ) reveals a degradation of the intensity profile. The parabolic pulse finally collapses.

To better understand the origin of this dramatic pulse change, let us consider the spectra recorded after 2, 3 and 4 maps (figure 3(c)). After three maps, the growth of unwanted bands of amplified regions becomes apparent. This can be interpreted as a manifestation of the scalar stimulated modulation instability (MI) which occurs in the anomalous segment [17] and which selectively amplifies bands of 
the parabolic broad spectrum. As spectral and temporal profiles are in the case of highly linear chirped pulses closely linked, the change of the spectral profile will directly translate into prohibitive temporal oscillations.
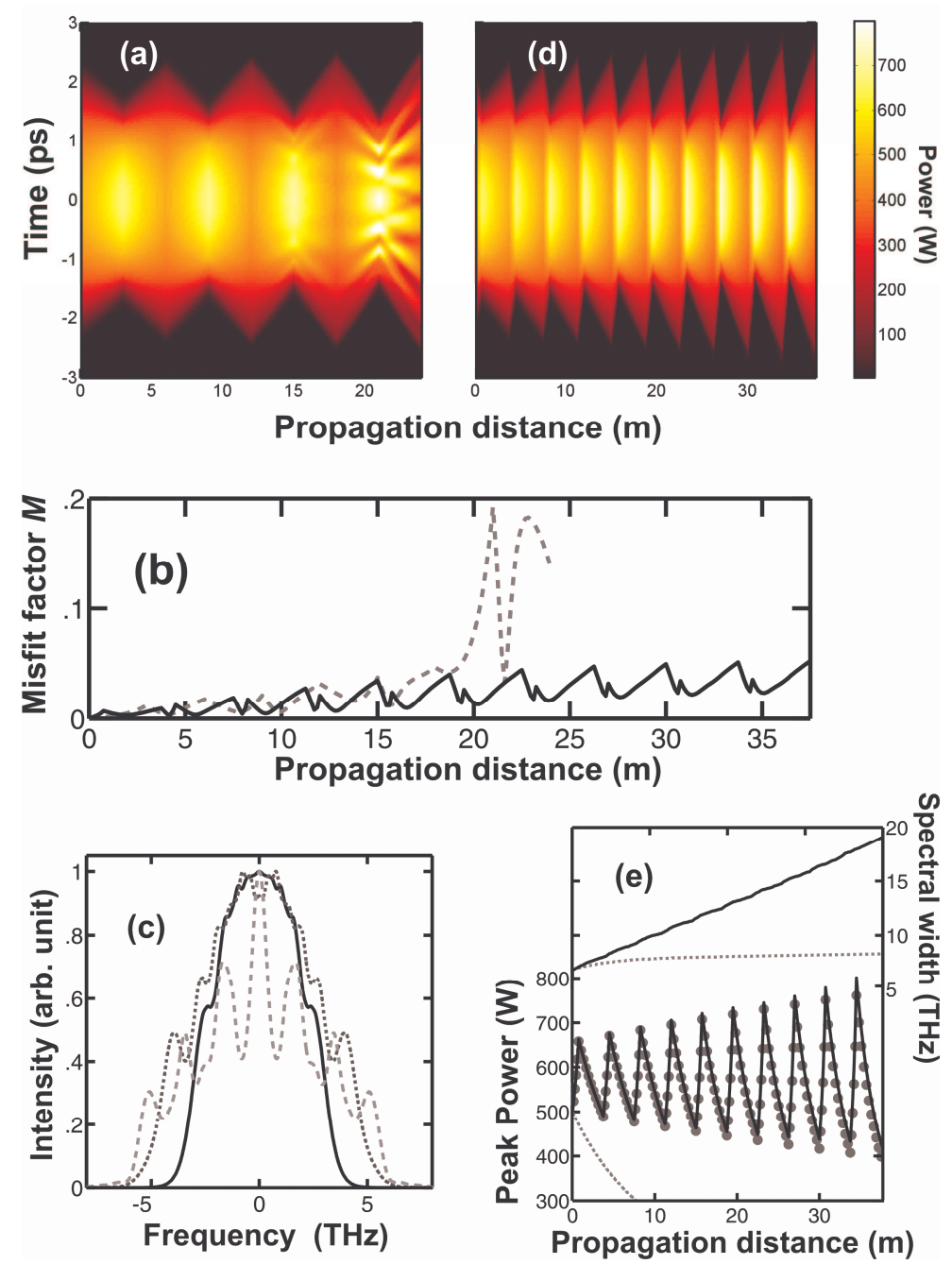

Figure 3 : (a) Longitudinal evolution of the temporal intensity over 4 maps made of fibres 1 and 2 (b) Longitudinal evolution of the misfit parameter for map 1 (dashed grey line) and for map 2 (solid black line) (c) Spectra after 2, 3 and 4 maps (solid line, dotted line and dashed grey line respectively). (d) Longitudinal evolution of the temporal intensity over 10 maps made of fibres 2 and 3. Longitudinal evolution of the peak power (e-left) and of the $-20 \mathrm{~dB}$ spectral width (eright) of a pulse evolving in a single normally dispersive fibre (fibre 2, grey dashed line) and in 10 maps made of fibres 2 and 3 (solid black line). Results derived from (7) are plotted with grey circles.

Even if MI can not be completely suppressed and will ultimately limit the propagation length, different solutions can be undertaken to minimize this detrimental issue. A first possibility could be to use a fibre with lower nonlinearity $\gamma$, which would lead to a lower MI gain. One can also use a fibre with higher dispersion (fibre 3, second order dispersion $\beta_{23}=4 \times \beta_{21}=-4010^{-3} \mathrm{ps}^{2} . \mathrm{m}^{-1}$ ). According to (9), this approach leads to a faster compression and then reduces the integrated MI gain. In order to maintain the same integrated dispersion, we have then used an anomalous dispersive segment with a fibre length reduced by a factor four (map 2) and we have tested this approach in figure 3(d). The propagation over 10 maps (and over up to 12 maps according to additional simulations not shown here) does not exhibit MI degradation. The parabolic shape is preserved, as it is confirmed in figure 3(b) with a misfit factor remaining as low as 0.05 after 10 maps. 
Also shown in figure 3(e) is the evolution of the peak power and spectral width. Equations (7) have been found excellent in predicting this evolution. The pulse undergoes a progressive nonlinear spectrum expansion, as it can also be seen in figure 4(a1). In a single normally dispersive fibre, due to the pulse broadening, the peak power of the pulse rapidly decreases (figure 3(e) bottom, dashed grey line), inducing less and less SPM, so that the spectral width saturates (figure 3(e) top, dashed grey line). In contrast, in the proposed dispersion-managed system, as the temporal broadening if periodically cancelled, the pulse keeps a high peak power at the input of the normally dispersive segment and self-phase modulation is thus cumulatively efficient. An increase of the spectral broadening by more than a factor 2 compared to a single normally dispersive fibre can be demonstrated (figure 3(e) top). Let us however note that in experimental situations, spectral broadening will be ultimately limited not by MI but by the impact of higher order effects such as splicing losses, third order dispersion (if the fibre dispersion is not flattened) or Raman scattering.

\section{Influence of the initial pulse shape and of the map average dispersion}

Let us now highlight the influence of the initial pulse profile. We compare in figure 4 the evolutions of initial parabolic and hyperbolic secant (sech) pulses. In both cases, the pulses undergo spectral broadening. But, whereas the parabolic pulse maintains its shape, the sech pulse develops a highly peaked spectrum with sharp edges. Details of the spectrum after 10 maps (figure 4(b)) illustrate the major distortions undergone by the spectral sech shape. This change also affects the temporal profile (figure 4(c)) with the development of a flattened pulse shape.
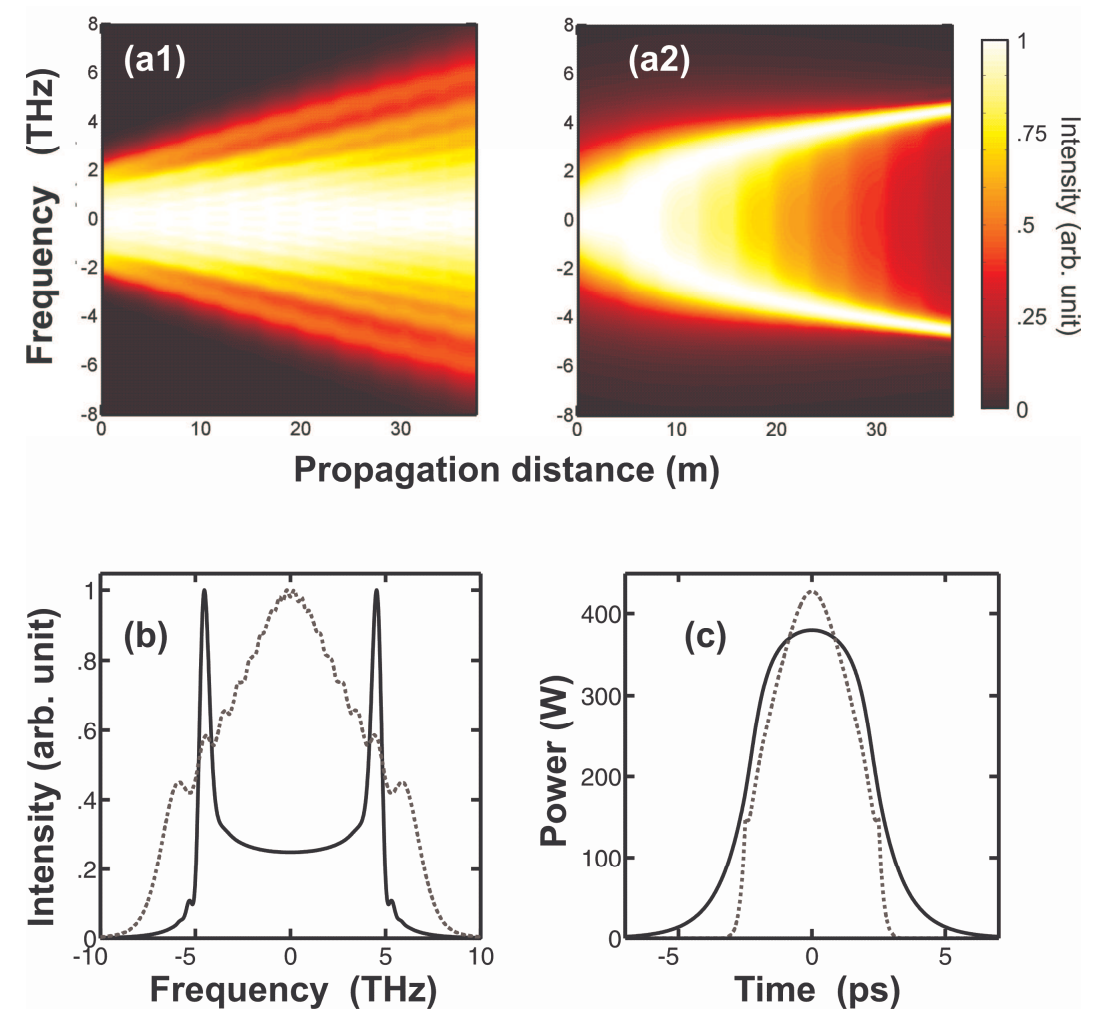

Figure 4 : (a) Longitudinal spectral evolution over ten maps made of fibres 2 and 3 for an initial parabolic pulse (a1) and an initial sech pulse (a2). Comparison of the output spectral (b) and temporal profiles (c). Sech and parabolic pulses are plotted with solid black lines and dotted grey line respectively. 
Finally, we have studied the impact of the integrated dispersion $L_{3} \beta_{23}+L_{2} \beta_{22}$ of a map. We have kept the length $L_{3}$ of the anomalous fibre (fibre 3) constant and we have varied the length $L_{2}$ of the normally dispersive segment (fibre 2). Evolution of the temporal width across the propagation in six maps is plotted in figure 5(a). For negative average dispersions, after each map, the pulse is further compressed, which is beneficial for spectral width and peak-power enhancement. However, after a given number of maps, the temporal width of the pulse becomes so short that problems mentioned in section 3 of the paper such as soliton compression will negatively act : the pulse will loose its characteristic parabolic features, as outlined by the high misfit factor observed in figure 5(a).

Let us now focus on the zero average dispersion case. We can notice that the breathing of the parabolic pulse is more and more pronounced after each map. This is mainly due to the continuous spectral broadening experienced by the pulse. Indeed, as the spectral width increases (as well as the chirp coefficient $C_{p}$ ), the compression stage in the anomalous fibre (equation (9)) is faster and faster. As a consequence, the peak power after the anomalous segment is higher and higher, as it can be seen on figure 3(e). With enhanced peak power and spectrum, the temporal broadening in the normally dispersive fibre will also be enhanced. If one targets output parabolic pulse with temporal width identical to the input, it is then desirable to use a slightly negative net dispersion. Regarding positive net dispersions, the pulse temporal broadening is higher, which seems beneficial for a low misfit factor $M$ (figure 5(c)).

From the curves simulated after 6,8 and 10 maps (figures $5(\mathrm{~b}, \mathrm{c})$ ), it is clear that higher the number of maps is, more pronounced will be the tendancies previously described.
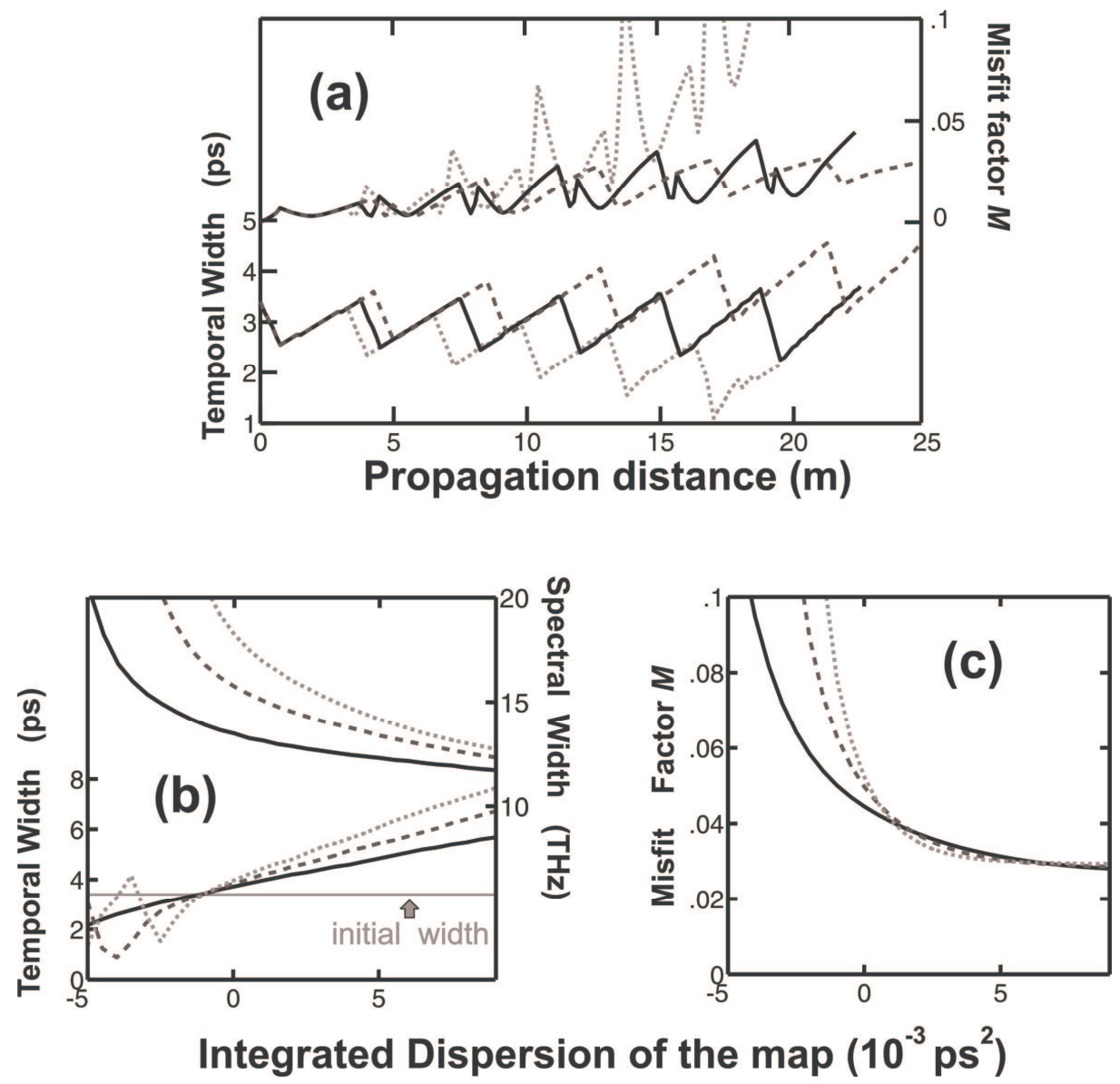

Figure 5 : Impact of the integrated dispersion of the map. Longitudinal evolution of the temporal width (a-bottom) and misfit factor (a-top) of the pulse for three values of integrated dispersion of the map $\left(-510^{-3}, 0\right.$ and $510^{-3} \mathrm{ps}^{2}$, dotted grey line, solid black line and dashed line respectively). Evolution of the output FWHM temporal width and spectral width (-20 dB width) (b) and of the misfit parameter (c) after 6, 8 and 10 maps (black solid, dashed and dotted grey lines respectively). 


\section{Conclusion}

In the present article, we have outlined the ability of a highly chirped parabolic pulse to maintain its shape through an arrangement of normally and anomalous dispersive fibres despite the presence of nonlinearity. By avoiding soliton compression and limiting MI deleterious impact, it is then possible to take advantage of the parabolic pulse ability to maintain its shape in normally dispersive fibres. By maintaining high peak-power, enhanced spectral broadening is then demonstrated. We expect this work to open new perspectives in the fields of coherent continuum generation as well as in the comprehension of the dynamics of pulses in self-similar fibre lasers [24]. Controlling the temporal spreading of a parabolic pulse will also be of practical interest in the context of telecommunication applications where pulse-to-pulse interactions can severely damage parabolic self-similar dynamics $[25]$.

\section{Acknowledgements}

We would like to thank Bertrand Kibler, Stéphane Pitois and Guy Millot for fruitful discussions. This research was supported by the Conseil Régional de Bourgogne and by the Agence Nationale de la Recherche (SUPERCODE project - ANR-06-BLAN-0401 as well as SOFICARS project - ANR-07RIB-013-03).

\section{References}

[1] Fermann M E, Kruglov V I, Thomsen B C, Dudley J M and Harvey J D 2000 Self-similar propagation and amplification of parabolic pulses in optical fibers Phys. Rev. Lett. 84 6010-3

[2] Dudley J M, Finot C, Millot G and Richardson D J 2007 Self-similarity in ultrafast nonlinear optics Nat. Phys. 3 597-603

[3] Anderson D, Desaix M, Karlson M, Lisak M and Quiroga-Teixeiro M L 1993 Wave-breakingfree pulses in nonlinear optical fibers J. Opt. Soc. Am. B 10 1185-90

[4] Parmigiani F, Finot C, Mukasa K, Ibsen M, Roelens M A F, Petropoulos P and Richardson D J 2006 Ultra-flat SPM-broadened spectra in a highly nonlinear fiber using parabolic pulses formed in a fiber Bragg grating Opt. Express 14 7617-22

[5] Dupriez P, Finot C, Malinowski A, Sahu J K, Nilsson J, Richardson D J, Wilcox K G, Foreman H D and Tropper A C 2006 High-power, high repetition rate picosecond and femtosecond sources based on Yb-doped fiber amplification of VECSELS Opt. Express 14 9611-6

[6] Billet C, Dudley J M, Joly N and Knight J C 2005 Intermediate asymptotic evolution and photonic bandgap fiber compression of optical similaritons around $1550 \mathrm{~nm}$ Opt. Express 13 3236-41

[7] Nicholson J W, Yablon A, Westbrook P S, Feder K S and Yan M F 2004 High power, single mode, all-fiber source of femtosecond pulses at $1550 \mathrm{~nm}$ and its use in supercontinuum generation Opt. Express 12 3025-34

[8] Finot C, Millot G, Pitois S, Billet C and Dudley J M 2004 Numerical and experimental study of parabolic pulses generated via Raman amplification in standard optical fibers IEEE J. Sel. Top. Quantum Electron. 10 1211-8

[9] Finot C, Provost L, Petropoulos P and Richardson D J 2007 Parabolic pulse generation through passive nonlinear pulse reshaping in a normally dispersive two segment fiber device Opt. Express 15 852-64

[10] Kibler B, Billet C, Lacourt P A, Ferrière R, Larger L and Dudley J M 2006 Parabolic pulse generation in comb-like profiled dispersion decreasing fibre Electron. Lett. 42 965-6 
[11] Finot C, Barviau B, Millot G, Guryanov A, Sysoliatin A and Wabnitz S 2007 Parabolic pulse generation with active or passive dispersion decreasing optical fibers Opt. Express 15 1582435

[12] Finot C, Parmigiani F, Petropoulos P and Richardson D J 2006 Parabolic pulse evolution in normally dispersive fiber amplifiers preceding the similariton formation regime Opt. Express 14 3161-70

[13] Finot C, Fatome J, Pitois S and Millot G 2007 All-Fibered High-Quality Low Duty-Cycle 20$\mathrm{GHz}$ and 40-GHz Picosecond Pulse Sources IEEE Photon. Technol. Lett. 19 1711-3

[14] Mollenauer L F, Mamyshev P V, Gripp J, Neubelt M J, Mamysheva N, Grüner-Nielsen L and Veng T 2000 Demonstration of massive wavelength-division multiplexing over transoceanic distances by use of dispersion-managed solitons Opt. Lett. 25 704-6

[15] Fatome J, Pitois S, Tchofo-Dinda P and Millot G 2003 Experimental demonstration of 160$\mathrm{GHz}$ densely dispersion-managed soliton transmission in a single channel over $896 \mathrm{~km}$ of commercial fibers Opt. Express 11 1553-7

[16] Agrawal G P 2001 Applications of nonlinear fiber optics (San Francisco, CA: Academic Press)

[17] Agrawal G P 2001 Nonlinear Fiber Optics, Third Edition (San Francisco, CA: Academic Press)

[18] Peacock A C 2001 Self-similar amplification and propagation of parabolic pulses in optical fibers. Master thesis at Auckland University, New-Zealand)

[19] International Telecommunication Union 2006 G.655 : Characteristics of a non-zero dispersion-shifted single-mode optical fibre and cable.

[20] Russell P S 2003 Photonic crystal fibers Science 299 358-62

[21] Finot C, Guenot A and Dupriez P 2007 Spectral compression of optical parabolic similaritons Ann. Phys. Fr 32 71-4

[22] Mollenauer L F, Stolen R H, Gordon J P and Tomlinson W J 1983 Extreme picosecond pulse narrowing by means of soliton effect in single-mode optical fibers Opt. Lett. 8 289-91

[23] Tomlinson W J, Stolen R H and Shank C V 1984 Compression of optical pulses chirped by self-phase modulation in fibers J. Opt. Soc. Amer. B 1 139-49

[24] Chong A, Renninger W and Wise F W 2007 Properties of normal-dispersion femtosecond fiber lasers J. Opt. Soc. Am. B 25 140-8

[25] Finot C and Millot G 2005 Interactions of optical similaritons Opt. Express 13 5825-30 